\title{
ANÁLISIS CRÍTICO DE LOS ESTILOS DE APRENDIZAJE DESDE UNA PERSPECTIVA NATURALISTA ${ }^{1}$
}

\author{
ULISES DELGADO SÁNCHEZ*, FERNANDA GABRIELA MARTÍNEZ FLORES**, DAVID JESÚS PONCE HERNÁNDEZ*** \\ UNIVERSIDAD AUTÓNOMA DEL ESTADO DE MORELOS, MÉXICO
}

Recibido: 29 de Abril de 2016

Aprobado: 12 de Julio de 2016

Para referenciar este artículo:

Delgado Sánchez, U., Martínez Flores, F. \& Ponce Hernández, D. (2016) Análisis crítico de los estilos de aprendizaje desde una perspectiva naturalista Revista Iberoamericana de Psicología: Ciencia y Tecnología, 9 (1), 45-52

\section{Resumen}

\begin{abstract}
Aunque el interés por el análisis empírico de los estilos de aprendizaje se ha incrementado durante las últimas dos décadas, la reflexión en torno a sus conceptos y supuestos es escasa. El presente escrito tiene por objetivo analizar la conceptualización actual de los estilos de aprendizaje, así como los instrumentos desarrollados para la evaluación de los mismos. Se cuestiona el estatus de variable explicativa de carácter multidimensional que se ha atribuido a los estilos de aprendizaje. Sustentados en la perspectiva interconductual de campo, se argumenta que estas concepciones caen en errores categoriales que limitan la aproximación teórica y metodológica a dichos eventos psicológicos, los cuales resultan ser especialmente relevantes en el proceso educativo. Ante las inconsistencias en la geografía lógica de los conceptos empleados para referirse a la noción de estilos de aprendizaje se propone considerar la teoría de campo interconductual para la evaluación y complementación de sus principales definiciones.

Palabras Clave: disposiciones interactivas, estilos de aprendizaje, estrategias de aprendizaje, universitarios.
\end{abstract}

\section{CRITICAL ANALYSIS OF LEARNING STYLES FROM A NATURALISTIC PERSPECTIVE}

\begin{abstract}
Interest in the empirical analysis of learning styles has increased over the past two decades; however, reflection on concepts and assumptions is scarce. The present paper aims to analyze the current conceptualization of learning styles as well as the developed instruments for evaluating them. The status of explanatory variable of multidimensional character, which has been attributed to learning styles, is questioned. Grounded on the inter-behavioral field perspective, it is argued that these conceptions fall into categorical errors that limit the theoretical and methodological approach to these psychological events, which are particularly relevant in the educational process. Given the inconsistencies in the logical geography of the concepts used to refer to the notion of learning styles, consideration of the formula from the inter-behavioral field theory for assessment and complementation of its main definitions it is proposed.

Key words: interactive dispossitions, learning styles, learning strategies, university
\end{abstract}

1 1Artículo realizado con el apoyo proporcionado por el PRODEP (DSA/103.5/14/7513) para el proyecto "Estilos de aprendizaje, competencias interactivas y logro académico en estudiantes universitarios de psicología de la UAEM".

* $\quad$ Profesor-Investigador de Tiempo Completo en el Centro de Investigación Transdisciplinar en Psicología de la Universidad Autónoma del Estado de Morelos, México. ulises.delgado@uaem.mx

** Maestra en diagnóstico y rehabilitación neuropsicológica. Estudiante dentro del Programa de Doctorado en Psicología de la UAEM (PNPC). maga.mtz@gmail.com

*** Maestro en Psicología. Estudiante dentro del Programa de Doctorado en Psicología de la UAEM (PNPC). lagarto43mx@ hotmail.com 
Diversos estudios han señalado que para lograr un aprendizaje efectivo es necesario considerar las necesidades, conocimientos, ideas previas y expectativas del estudiante, así como también sus estilos y estrategias de aprendizaje (Beltrán, 2003; Bolívar y Rojas, 2008; Díaz et al. 2009; Lugo, Rodríguez y Montijo, 2012). De ahí que en las últimas décadas se ha incrementado el interés por el análisis de los procesos educativos, específicamente aquellos involucrados en el aprendizaje y los factores que en él influyen.

Lo anterior se ha visto reflejado en las numerosas publicaciones que han aparecido en los últimos años acerca de los estilos de aprendizaje, sobre todo aquellos que se pueden identificar en estudiantes de bachillerato y universitarios. Dichas investigaciones se han dirigido principalmente al análisis de los perfiles de estilos con relación a diversas variables, como contenidos, metodologías didácticas, exigencias de cada carrera, nivel cognitivo, características afectivo-motivacionales, contextuales y de género asociadas al logro académico (Bahamón, Vianchá, Alarcón y Bohórquez, 2012; Martín, 2011). Aun con la amplia investigación sobre estas variables y su relación con el aprendizaje y el éxito académico, su conceptualización se ha realizado principalmente desde la postura cognoscitivista, por lo que son identificables los sesgos interpretativos que atribuyen facultades explicativas a los estilos de aprendizaje, en lugar de concebirlos como factores potencializadores del aprendizaje escolar.

Es importante mencionar que la concepción del estilo de aprendizaje influye necesariamente en la forma en que se tipifica, analiza y evalúa. Por tal motivo, el propósito de este artículo es realizar una revisión crítica de la noción de estilos de aprendizaje desde una perspectiva interconductual de campo (Kantor, 1980), misma que permite considerar el papel de la historia interactiva como factor clave para explicar las consistencias individuales en el proceso de aprendizaje, a las cuales se refiere legítimamente.

En primera instancia se abordarán dos de las definiciones más importantes de estilos de aprendizaje, la propuesta por Keefe (1988) y la de Kolb (1981, 1984); así como los principales instrumentos de evaluación derivados de ellas. Se realiza un análisis crítico que pone de manifiesto algunos errores categoriales (Ryle, 1967) e inconsistencias teóricas que el enfoque interconductual permite identificar. Asimismo, se realizará una propuesta de análisis de los estilos de aprendizaje caracterizándolos como estilos interactivos, para finalmente argumentar la utilidad del uso de los arreglos contingenciales en la evaluación de las consistencias individuales en el proceso de aprendizaje (Ribes, 1990).

\section{Análisis del concepto de estilos de aprendizaje}

A partir de 1950, el término de estilos de aprendizaje fue utilizado en el marco de la teoría cognoscitiva. Concebidos principalmente como estilos cognitivos, se consideraba que expresaban las formas particulares de los individuos de percibir y procesar la información. Más tarde, en contraparte con los teóricos de la personalidad, los psicólogos educativos mostraron preferencia por el término estilos de aprendizaje, considerándolo un término explicativo del carácter multidimensional del proceso de adquisición de conocimientos (Cabrera, 2003).

Se ha destacado que este concepto está estrechamente relacionado con dimensiones psicológicas tales como la personalidad y la capacidad cognitiva (Carvajal, Trejo y Barros, 2007), así mismo se ha vinculado a los estilos de aprendizaje con el fracaso o aprovechamiento académico, la formación académica, el género (Juárez, Rodríguez, y Luna, 2012), el campo disciplinar al que pertenece el estudiante y cohorte generacional (Labatut, 2005; Laguzzi, Bernardi, Araujo, Ventura \& Vigliano, 2013; Bahomón, Vianchá, Alarcón y Bohórquez, 2012). Otros autores exponen hallazgos divergentes a los primeros, sobre todo en los cohortes generacionales (Ordóñez, Rosety-Rodríguez, y Rosety-Plaza, 2003), así como el sexo (Esguerra y Guerrero, 2010; Labatut, 2005; López y Ballesteros, 2003).

En la actualidad el desarrollo teórico ha llevado a la construcción de una gran variedad de definiciones de estilos de aprendizaje, una de las más importantes es la de J. W. Keefe (citado por Alonso, Gallego y Honey, 1994), quien los describe como: "comportamientos cognitivos, afectivos y psicológicos característicos que sirven como indicadores relativamente estables, de cómo los alumnos perciben, interactúan y responden al ambiente de aprendizaje" (p.48).

En esta definición, es notable la falta de precisión sobre cuáles son los procesos cognitivos a que se hace referencia, si a todos o a algunos de ellos, como la percepción, la atención, la memoria, el razonamiento, etc. En cuanto a los comportamientos afectivos, no se especifica si este elemento puede ser entendido como la disposición que tiene el individuo ante la ejecución de una tarea, los aspectos motivacionales ante la misma, su estado de ánimo durante esta, su rechazo o aceptación de la tarea. Lo que pone de manifiesto el carácter ambiguo de la definición y la perspectiva sobre la cual se conciben las consistencias en la forma de responder ante el ambiente.

La segunda definición con gran influencia entre los estudiosos de los estilos de aprendizaje es la de Kolb (1984), quien incluye el concepto dentro de su modelo 
de aprendizaje experiencial, definiéndolo como: “Algunas habilidades de aprender, que se destacan por encima de otras, como resultado del aparato hereditario, de las experiencias particulares de vida y de las exigencias del medio ambiente actual o del entorno" (p.56).

En esta propuesta sobre estilos de aprendizaje se señala la falta de especificidad sobre el concepto de aparato hereditario, puesto que no se menciona si se refiere a la herencia genética o al aspecto hereditario de la cultura que evoluciona con el tiempo y se transmite por medio de la sociedad. Asimismo es importante destacar que la definición de Kolb considera las experiencias particulares de vida de los alumnos; sin embargo no se sabe si toma en cuenta únicamente el conocimiento adquirido en las vivencias o también la influencia de la interacción.

Con base en las definiciones anteriores se deja en claro que si bien el término "estilo" se ha desarrollado en diferentes áreas como la personalidad, la comunicación, la motivación, el comportamiento y el aprendizaje, en todas ellas se hace referencia a preferencias en la manera de aprender en ciertas etapas de la vida, vinculadas a la base psicológica y fisiológica de la individualidad, así como a las características del ambiente. De igual forma, se reconoce que los estilos de aprendizaje tienen flexibilidad y posibilidad de cambiar o reajustarse según las exigencias del medio para el logro de un aprendizaje más eficiente (González, 2011). Aunque ello no explica por qué los individuos con una formación semejante y oportunidades similares de aprendizaje reaccionan de forma diferente ante aquello que deben aprender.

\section{Análisis de los instrumentos de evaluación de los estilos de aprendizaje}

Las definiciones propuestas por Keefe (1988) y por Kolb (1984) han dado origen a tres de los principales instrumentos de evaluación de los estilos de aprendizaje. 1) el Index of Learning Style (ILS) de Felder \& Silverman (1988), quizá el más ampliamente utilizado (Solomon \& Felder, 2001; Troiano, Breitman y Gete-Alonso, 2004; Ventura, 2011; Ventura, Moscoloni \& Gagliardi, 2012); 2) el Learning Style Inventory (LSI), derivado del modelo de Kolb (1981); y 3) el Cuestionario Honey-Alonso de Estilos de Aprendizaje (CHAEA) de Alonso, Gallego y Honey (1994) con mayor presencia en España y en América Latina (Carvajal, Trejos y Barros, 2007; Esguerra y Guerrero, 2010; Juárez, Rodríguez y Luna, 2012; Ventura, 2011).

El ILS se basa en la definición original de Keefe (1988), y se compone de 44 preguntas que permiten apreciar la preferencia de las cuatro dimensiones del modelo del Felder y Silverman (citado por Felder \& Spurlin, 2005). Este modelo clasifica los estilos de los estudiantes en las siguientes cuatro dimensiones.

a) Percepción (Sensorial/intuitivo). El estudiante sensorial tiende a ser un pensador concreto, práctico, orientado hacia hechos y procedimientos; el intuitivo es un pensador abstracto, innovador, orientado hacia las teorías y significados subyacentes.

b) Representación (Visual/Verbal). El estudiante visual prefiere las representaciones visuales del material presentado, como imágenes diagramas y gráficos de flujo; el verbal se inclina más a los aspectos escritos y prefiere explicaciones habladas.

c) Procesamiento (Activo/Reflexivo). El estudiante activo aprende por medio de probar cosas y disfruta trabajando en grupos; el reflexivo aprende por medio de pensar las cosas, prefiere trabajar solo o con un único compañero.

d) Comprensión (Secuencial/Global). El estudiante secuencial tiene un proceso de pensamiento lineal, aprende en pequeños pasos; el global tiene un procesamiento de pensamiento holístico y aprende en grandes saltos.

Existe poca claridad en torno a estas cuatro dimensiones. Un ejemplo es que la dimensión de percepción podría considerarse como comportamiento cognitivo o como afectivo, pues no se establece qué se entiende por uno u otro. Esta dimensión es confusa, ya que al referirse al pensamiento concreto en el tipo sensorial y al pensamiento abstracto en el tipo intuitivo no se delimita la posible combinación de ambos tipos de pensamiento para el logro de una tarea.

Tampoco se sabe si la dimensión de representación (visual/verbal) pertenece a la forma en que el alumno interactúa o a cómo percibe, ya que falta explicar si se refiere al sentido por el cual se percibe la información, visual o auditiva, o bien a aspectos relacionados con la teoría del cerebro derecho vs cerebro izquierdo (Velásquez, Remolina y Calle, 2007). En dicho caso se estaría haciendo alusión a las funciones del hemisferio derecho con una predominancia de procesamiento de imágenes 
y a las del izquierdo con inclinación hacia el manejo del lenguaje oral o escrito. Al no esclarecerse estas cuestiones, la definición es insuficiente.

En lo que respecta a la dimensión de procesamiento (activo/reflexivo), esta se apega a la teoría cognoscitiva, pero retoma aspectos ajenos a ella al considerar la inclinación por probar las cosas y el trabajo en grupo en el caso del activo, y la preferencia por el trabajo individual en el reflexivo. Hace referencia a aspectos de la interacción del alumno con el ambiente de aprendizaje que podrían relacionarse con teorías de la personalidad como la de los cinco grandes rasgos de Goldberg (1992). Tal es el caso de los rasgos de apertura a la experiencia y extroversión en la categoría activo, y el rasgo introversión en el reflexivo.

En la última dimensión, comprensión (secuencial/global), se retoman las teorías del funcionamiento cerebral sin esclarecer si la comprensión es considerada como comportamiento cognitivo y si el tipo de comprensión en el estudiante tiene alguna relación con lo postulado por la teoría del cerebro triuno (Velásquez, Remolina y Calle, 2007), donde la predominancia del hemisferio izquierdo explica con su función de análisis-síntesis el carácter secuencial del pensamiento, y la predominancia del derecho la capacidad de abstraer globalidades. Aun con la ambigüedad en estas categorías podemos identificar el intento de considerar diversos factores relacionados con el aprendizaje, como los aspectos fisiológicos, perceptivos, cognitivos y someramente aspectos interactivos.

El instrumento Learning Style Inventory (LSI) propuesto por Kolb (1981), (Romero, Salinas, y Mortera, 2010), mide la diferencia de estilos de aprendizaje en dos dimensiones: 1) percepción del medio: abstracto-concreto y 2) procesamiento: activo-reflexivo. Se basa en cuatro habilidades que el aprendizaje requiere: la habilidad en experiencia concreta (CE), en observación reflexiva (RO), en conceptualización abstracta (AC) y en experimentación activa (AE).

De la combinación de las dimensiones se toman cuatro estilos que son:

a) Convergente: Los estudiantes dominan las habilidades AC y AE; tienden a emplear el razonamiento hipotético-deductivo para centrarse en un problema específico.

b) Divergente: Tienen buen desempeño en la EC y OR; su mayor fuerza radica en la capacidad imaginativa.

c) Asimilador: Las habilidades de aprendizaje que dominan son AC y OR; su mayor fuerza radica en la capacidad de crear modelos teóricos. d) Acomodador: Los estudiantes se destacan en $\mathrm{EC}$ y $\mathrm{AE}$; su mayor fuerza radica en hacer las cosas, realizar planes y experimentos, y participar en nuevas experiencias.

La anterior propuesta de evaluación, basada en la definición de Kolb (1981), señala que las dimensiones percepción del medio y procesamiento, están vinculadas con los aspectos cognitivos, sin embargo no se encuentran plasmadas directamente en la definición. Dentro de estas dimensiones de índole cognitivo se encuentran cuatro habilidades que Kolb (1981), considera importantes para el aprendizaje: la habilidad CE, los estudiantes son capaces de involucrarse plena y abiertamente, y sin parcialidades a las nueva experiencias; CA, son capaces de reflexionar y observar sus experiencias desde muchas perspectivas; $\mathrm{RO}$, son capaces de crear conceptos que integren sus observaciones dentro de teorías lógicas; y EA, en la que deben ser capaces de usar las teorías para tomar decisiones y resolver problemas. Si bien estas habilidades forman parte del proceso de aprendizaje experiencial que propone Kolb (1981), el cual busca integrar el paradigma cognoscitivo y conductual para explicar las diferencias individuales en el aprendizaje, se observa un vacío en cuanto al papel de la historia interactiva que enmarca a la experiencia del individuo y su relación con la forma de desempeñarse ante las exigencias del medio. Un aspecto notable del modelo de Kolb (1981), es que procura destacar en sus categorías la historia de aprendizaje del estudiante, que aunque es parcial respecto a toda su historia interconductual, permite valorar la importancia del comportamiento histórico respecto al proceder presente.

Por otro lado, el cuestionario de Honey-Alonso (Alonso, Gallego y Honey, 1994), evalúa cuatro estilos de aprendizaje: activo, reflexivo, teórico y pragmático.

a) Activo: Predomina la implicación en las experiencias, tiene preferencia por resultados inmediatos, así como al diálogo y al debate.

b) Reflexivo: Tendencia a considerar sus experiencias y observarlas desde diferentes perspectivas, tiende a reunir datos y analizarlos para llegar a conclusiones.

c) Teórico: Enfoca los problemas por etapas lógicas, analiza, sintetiza, busca la racionalidad y la objetividad.

d) Pragmático: Aplicación práctica de las ideas y su experimentación, toma decisiones con rapidez (Alonso. y Gallego, 2004; Aragón y Jiménez, 2009; Castro y Guzmán, 2005). 
Alonso, Gallego y Honey (1994), en la construcción del CHAEA, parten de los planteamientos de Honey \& Mumford (1986), quienes a su vez se sustentan en lo propuesto por Kolb (1984). El planteamiento de esta evaluación, a diferencia de lo postulado originalmente por Kolb (1984), homologa los procesos a cuatro estilos independientes simplificando el modelo. Sin embargo, Freiberg y Fernández (2013) mencionan la inconsistencia teórica y empírica en el análisis de la calidad psicométrica del instrumento; pues al realizar un análisis correlacional entre los estilos se encuentran fuertes asociaciones entre los mismos. Al no ser excluyentes entre sí, los cuatro estilos fracasan al reproducir la solución factorial que proponen los autores en su modelo teórico.

Puede apreciarse que la conceptualización de los estilos de aprendizaje, así como las dimensiones que consideran los principales instrumentos de evaluación, caen en errores categoriales al emplear en sus constructos términos que pueden entrar en categorías lógicas diferentes. La forma en la que son evaluados no permite observar cómo es la ejecución de las habilidades de aprendizaje, la manifestación conductual de las dimensiones evaluadas y la manera en la que los estudiantes responden a las exigencias del medio.

La conceptualización y evaluación de los estilos de aprendizaje puede enriquecerse si se toma en cuenta la historia interactiva de los individuos, permitiendo así observar la emergencia de la historia de interacciones, como consistencias individuales, ante las diversas condiciones del entorno académico.

\section{Propuesta de análisis interconductual de los estilos de aprendizaje}

Como se ha expuesto en las secciones precedentes, el análisis de los estilos de aprendizaje ha cobrado importancia considerable en la investigación educativa. Ello no es casual, pues parece deberse a la reconsideración de la importancia de las variables históricas que conforman la respuesta individual ante las tareas de aprendizaje escolar. Como es común en la historia de nuestra disciplina, las aproximaciones conceptuales y metodológicas al inicio no convergen con la complejidad del objeto de estudio, respaldando su avance en definiciones operacionales más que en definiciones funcionales.

El interconductismo postula que el objeto de estudio de la psicología es la interacción funcional de los individuos con respecto a objetos o eventos estimulantes. Desde este punto de vista, la psicología educativa debe hacer especial énfasis en los episodios de interacción entre los estudiantes y las tareas, objetos y procesos que tienen lugar en los contextos educativos, considerando que las mismas desarrollan continuamente una historia que determina en gran manera las interacciones futuras (Kantor, 1980). Las categorías del modelo interconductual constituyen una plataforma sólida para el análisis de los estilos de aprendizaje, en tanto episodios psicológicos.

$E P=C(k, f r, f e, h i, f d, m d)$, en donde la función de respuesta (fr) se identifica como una actividad del organismo, la función de estímulo (fe) como una actividad del objeto estimulante, el proceso histórico interconductual (hi) el cual se ha desarrollado a través de una serie de contactos del organismo con los objetos, los factores disposicionales (fd) que consisten en las circunstancias inmediatas que influyen en la $\mathrm{fe} \leftrightarrow \mathrm{fr}, \mathrm{y}$ el medio de contacto o interconductual (md). Cabe mencionar que en esta fórmula la unicidad de los campos interconductuales se simboliza con la $k$, y la $C$ indica que el campo consistente es un sistema de factores de interacción. (Kantor, 1980, p.27),

Sustentamos que los estilos de aprendizaje pueden ser comprendidos de mejor manera si se considera su carácter disposicional (Delgado, 1999). Ryle (1967) menciona que: "poseer una propiedad disposicional no consiste en encontrarse en un estado particular o experimentar determinado cambio. Es ser susceptible de encontrarse en un estado particular o de experimentar un cambio cuando se realiza determinada condición" (p.43). Así, los estilos de aprendizaje pueden entenderse como capacidades o propensiones que describen probabilidades de acción en condiciones específicas, y no como acciones u ocurrencias que pueden ser observables mediante la aplicación de test estandarizados.

Las categorías disposicionales de la teoría de la conducta permiten dar cuenta de los factores históricos que influyen de forma predictiva con relación a los estímulos y las respuestas (Delgado y lbarra, 2001). Dentro de estas categorías, Ribes (1990) incluye el concepto de estilos interactivos, los cuales son la manera consistente en que el individuo interactúa funcionalmente en una situación donde no se establecen o se exigen determinados modos de comportamiento, dicho de otro modo, no se especifican criterios de logro. Aunado a esto, este autor postula que las situaciones de interacción en las que participan los individuos pueden concebirse como arreglos contingenciales, que permiten el estudio de la relación funcional entre el individuo y los elementos del medio, es decir, de los estilos interactivos como interacción (Rodríguez, 2010).

Este aporte permite enriquecer la evaluación y categorización de los estilos de aprendizaje, puesto que la exis- 
tencia o no de un logro en el arreglo contingencial (criterio cerrado o abierto), permite por un lado dar respuesta a la capacidad de ajuste a la situación y por ende a la competencia, y por el otro permite obtener información sobre la forma de respuesta que subyace a su historia interactiva, es decir, a la dimensión disposicional de los estilos de aprendizaje de los estudiantes. Ribes (2009) plantea que el comportamiento de los individuos no puede evaluarse como un mero inventario reactivo de respuestas indirectas a una supuesta variable o basándose en morfologías de respuesta. Por el contrario, el comportamiento del individuo debe medirse como una interacción funcional, en tiempo real, con los eventos, propiedades y relaciones que configuran una situación definida contingencialmente.

Desde este enfoque de estudio puede enriquecerse la noción de estilos de aprendizaje, al conceptualizar la diferencia entre individuos como un indicador secundario de las consistencias intraindividuales en el comportamiento y como constancias en las que el individuo constituye su propio marco de comparación (Ribes, 2009).

\section{Conclusiones}

Las principales conceptualizaciones y propuestas de evaluación sobre el concepto de estilos de aprendizaje que se han presentado y analizado en este trabajo, han tenido gran relevancia en el auge de la investigación sobre cómo los estudiantes enfrentan las situaciones de aprendizaje en diferentes contextos educativos. Pese a esto se observan inconsistencias teóricas provenientes de la presencia de errores categoriales que obstaculizan su poder descriptivo y explicativo, así como la metodología empleada para la evaluación de este constructo.

Este artículo ha presentado y analizado las definiciones más influyentes y los instrumentos más usados en el análisis de los estilos de aprendizaje. Los enfoques revisados parten de la teoría cognoscitivista, cuyos principios plantean la existencia de procesos y conciben su ubicación orgánica, reduciendo al individuo a los aspectos cognitivos de la mente, y apuntando a la racionalización del comportamiento de la persona al enfrentar el aprendizaje.

Asimismo concibe tácita o expresamente a los individuos como simples agentes que responden de determinada manera ante un evento, debido a aspectos hereditarios, psicológicos, afectivos y fisiológicos de forma mecánica, limitando las posibilidades de acción de las personas ante las contingencias y anulando las disposiciones interactivas que son parte de la historia de los individuos.

Las disposiciones de un estudiante para interrelacionarse con el aprendizaje no pueden ser limitadas a los aspectos cognitivos, ni reducir a actos verbales su evaluación, caso de los instrumentos de evaluación CHAEA, ILS, y LSI, que aplican preguntas para determinar los estilos de los estudiantes, cuando estos son manifestaciones conductuales más amplias, complejas y difusas. Este tipo de evaluación deja de lado al estudiante y su relación con el medio; corresponden a posturas deterministas que postulan que el estilo del estudiante determina su comportamiento ante el aprendizaje, dejando de lado el poder de cambio que tienen en la ejecución de tareas, según la naturaleza de las mismas, y omitiendo así las contingencias. Estos instrumentos no consideran la observación del estudiante al momento de que se le plantea la ejecución de una tarea o la solución de un problema; dejan de lado el proceso de aprendizaje en el momento que se efectúa y plantean únicamente enunciados hipotéticos que se contestan con respuestas predeterminadas, dejando de lado la libre expresión del estudiante.

Parte de la propuesta alternativa que resulta de este análisis crítico, se señalan las oportunidades de desarrollo teórico mediante el uso de arreglos contingenciales para el estudio de los estilos interactivos y las consistencias individuales en el proceso de aprendizaje. De este modo, al tomar en cuenta la historia interconductual para referirse a los eventos psicológicos, se da la pauta para subsanar los vacíos que deja la falta de delimitación conceptual en cuanto a si el estilo de aprendizaje encuentra su raíz fundamental en el individuo o en el ambiente. Es así que la crítica de las propuestas de definición y evaluación revisadas, permite la complementación y reformulación de los conceptos que componen la noción de estilos de aprendizaje, dejando a la investigación el encargo de verificar su utilidad y poder explicativo.

Por ello es necesario comenzar a replantear las maneras en que se conciben y evalúan estos aspectos, permitiendo así realizar investigaciones que no solamente arrojen aspectos descriptivos sino predictivos del comportamiento, en este caso, de los estudiantes. Las investigaciones que se han realizado se han centrado en dar explicaciones sobre cómo se relacionan los estilos de aprendizaje con otras variables, en lugar de tratar de predecir el comportamiento de los individuos, que son los agentes en los que se centra el aprendizaje; dejando de lado con ello la interacción de los individuos con el objeto de aprendizaje. Esta postura permitiría observar la manifestación de los estilos de aprendizaje en diferentes contextos y ampliar la visión sobre el papel de la interacción entre el individuo, las disposiciones, su historia interactiva y las exigencias del medio. 


\section{Referencias}

Alonso, C., y Gallego, D. (2004). Los Estilos de Aprendizaje: una propuesta pedagógica. I Congreso Internacional de estilos de aprendizaje. Madrid.

Alonso, C., Gallego D., y Honey, P. (1994). Los Estilos de Aprendizaje: Procedimientos de diagnóstico y mejora. Bilbao: Ediciones Mensajero.

Aragón, M., y Jiménez, Y. (2009). Diagnóstico de los estilos de aprendizaje en los estudiantes: Estrategia docente para elevar la calidad educativa. Revista de Investigación Educativa, 9, 1-21.

Bahamón, M., Vianchá, M., Alarcón, L., y Bohórquez, C. (2012). Estilos y estrategias de aprendizaje: una revisión empírica y conceptual de los últimos diez años. Pensamiento Psicológico, 10 (1), 129-144.

Beltrán, J. (2003). Estrategias de aprendizaje. Revista de educación. 332, 55-73

Bolívar, J., y Rojas, F. (2008). Los estilos de aprendizaje y el locus de control en estudiantes que inician estudios superiores y su vinculación con el rendimiento académico. Investigación y Postgrado, 23 (3), 199-215.

Cabrera, J. (2003). La comprensión del aprendizaje desde la perspectiva de los estilos de aprendizaje. Pinar del Rio: Universidad Hermanos Sainz.

Castro, S., y Guzmán, B. (2005). Los estilos de aprendizaje en la enseñanza y el aprendizaje: una propuesta para su implementación. Revista de Investigación, 58, 83-102.

Carvajal, P., Trejos, A., y Barros, A. (2007). Análisis estadístico multivariado de los estilos de aprendizaje predominantes en estudiantes de Ingenierías de la Universidad Tecnológica de Pereira, $2^{2}$ semestre de 2006, Scientia et Technica, 13(34), 325-330.

Delgado, U. (1999) ¿Qué son las preferencias cognitivas? Alternativas en Psicología, IV(6), 33-37.

Delgado, U., y Ibarra, L. R. (2001). ¿Son las preferencias cognoscitivas disposiciones psicológicas? Alternativas en Psicología, VI(7), 1-6.

Díaz, G., Mora, S., Lafuente, J., Gargiulo, P., Bianchi, R., Terán, C., Gorena, D., Arce, J., y Escanero, J. (2009). Estilos de aprendizaje de estudiantes de medicina en universidades latinoamericanas y españolas: relación con los contextos geográficos y curriculares. Educación Médica, $12,183-194$.

Esguerra, G., y Guerrero, P. (2010). Estilos de aprendizaje y rendimiento académico en estudiantes de psicología. Diversas Perspectivas Psicológicas, 6 (6), 97-109.

Felder,R.M., \& Silverman, L. K. (1988). Learning and teaching styles in engineering education. Engr. Education, 78 (7), 674-681.

Felder, R., \& Spurlin, J. (2005). Applications, Reliability and Validity of the Index of Learning Styles. International Journal of Engineering Science, 1(21), 103-112.
Freiberg, A., y Fernández, M. (2013). Cuestionario Honey-Alonso de estilos de aprendizaje: Análisis de sus propiedades Psicométricas en Estudiantes Universitarios. Summa Psicológica UST, 10(1), 103-117.

Goldberg, L. R. (1992). The development of markers of the Big-Five factor structure. Psychological Assessment, 4, 26-42.

González, M. (2011). Estilos de aprendizaje: su influencia para aprender a aprender. Revista Estilos de Aprendizaje, 7(7) 1-13.

Honey, P., y Mumford, A. (1986). Using your Learning Styles $2^{\underline{a}}$ ed. Berkshire: Maidenhead.

Juárez, C., Rodríguez, G., y Luna, E. (2012). El cuestionario de estilos de aprendizaje CHAEA y la escala de estrategias de aprendizaje ACRA como herramienta potencial para la tutoría académica. Revista Estilos de Aprendizaje, $10(10), 1-31$.

Kantor, J. R. (1980). Psicología interconductual. Un ejemplo de construcción científica sistemática. México: Trillas.

Keefe, J. (1988). Profiling and Utilizing Learning Style. Virginia: NASSP.

Kolb, D. (1981). Learning styles and Disciplinary Differences. The Modern American Collage. United States of America.

Kolb, D. (1984). Experiential Learning. Experience as the source of learning and Development. Nueva Jersey: Prentice Hall.

Labatut, E. (2005). Evaluación de los estilos de aprendizaje y metacognición en estudiantes universitarios. Revista de Psicopedagogía, 6(22), 1-17.

Laguzzi, J., Bernardi, S., Araujo, A., Ventura, A., y Vigliano, F. (2013). Estilos de aprendizaje de los estudiantes de medicina veterinaria de la Universidad Nacional de Rosario, Argentina. Revista Veterinaria 24(2), 151-156.

López, C., y Ballesteros, B. (2003). Evaluación de los estilos de aprendizaje en estudiantes de enfermería mediante el cuestionario CHAEA. Enfermería Global, 3, 1-12.

Lugo, J., Rodríguez, C., y Montijo, L. (2012). El cuestionario de estilos de aprendizaje CHAEA y la escala de estrategias de aprendizaje como herramienta potencial para la tutoría académica. Revista Estilos de Aprendizaje, 10(10), 1-31.

Martín, A. (2011). Competencias del estudiante autorregulado y los estilos de aprendizaje. Revista de Estilos de Aprendizaje, 8(8), 1- 17.

Ordóñez, F., Rosety-Rodríguez, M., y Rosety-Plaza, M. (2003). Análisis de los estilos de aprendizaje predominantes entre los estudiantes de ciencias de la salud. Revista de Enfermería Global. 3, 1-6.

Ribes, E. (1990). Problemas conceptuales en el análisis del comportamiento. México: Trillas.

Ribes, E. (2009). La personalidad como organización de los estilos interactivos. Revista Mexicana de Psicología, 26(2), 145-161. 
Rodríguez, M. (2010). Estrés: una propuesta naturalista. SUMMA Psicológica UST, 7(1), 51-62.

Romero, L., Salinas, V., y Mortera, F. (2010). Estilos de aprendizaje basado en el modelo de Kolb en la educación virtual. Revista de Innovación Educativa, 2 (1).

Ryle, G. (1967). El concepto de lo mental. Buenos Aires: Paidós.

Solomon, B., \& Felder, R. (2001). Index of Learning Styles Questionnaire. North Carolina State University.

Troiano, H., Breitman, M., y Gete, C. (2004). Estilos de aprendizaje que predominan entre los estudiantes universitarios. Revista Enseñanza Universitaria, 23, 23-82.

Velásquez, B. Remolina, N., y Calle, M. (2007). Determinación del perfil de dominancia cerebral o formas de pensamiento de los estudiantes de primer semestre del programa de bacteriología y laboratorio clínico de la Universidad Colegio Mayor de Cundinamarca. Nova, $7(5), 48-56$.

Ventura, C. (2011). Estilos de aprendizaje y prácticas de enseñanza en la universidad. Un binomio que sustenta la calidad educativa. Perfiles Educativos, 33.

Ventura, C., Moscoloni, N., y Gagliardi, R. (2012). Estudio comparativo sobre los estilos de aprendizaje de estudiantes universitarios argentinos de diferentes disciplinas. Psicología desde el Caribe, 29(2), 276-304. 